\title{
Feasibility of using point-of-care lung ultrasound for early triage of COVID-19 patients in the emergency room
}

\author{
Nick Narinx ${ }^{1}$ (1) $\cdot$ Annick Smismans ${ }^{2} \cdot$ Rolf Symons $^{3} \cdot$ Johan Frans ${ }^{2} \cdot$ Annick Demeyere $^{3} \cdot$ Marc Gillis $^{4}$
}

Received: 14 June 2020 / Accepted: 31 August 2020 / Published online: 10 September 2020

(C) American Society of Emergency Radiology 2020

\begin{abstract}
Purpose Diagnostic value of point-of-care lung ultrasound (POCUS) in detection of coronavirus disease (COVID-19) in an emergency setting is currently unclear. In this study, we aimed to compare diagnostic performance, in terms of sensitivity, specificity, positive predictive value (PPV), negative predictive value (NPV), and accuracy, of POCUS lung, chest CT, and RT-PCR for clinically suspected COVID-19 infections in patients submitting to the emergency room (ER).

Material and methods This retrospective study enrolled 93 patients with a suspected COVID-19 infection, admitted to the ER between March 28th and April 20th, 2020. Test subjects showed one or more symptoms of an acute respiratory infection, for which consequent COVID-19 testing was achieved using POCUS lung, chest CT, and RT-PCR. CT images were analyzed by 2 radiologists blinded to RT-PCR results. POCUS lung was performed by three emergency medical doctors, and reports were analyzed by the researcher, blinded to clinical information, US imaging, CT, and RT-PCR test results.

Results Compared with RT-PCR, POCUS lung demonstrated outstanding sensitivity and NPV ( $93.3 \%$ and $94.1 \%$ respectively) while showing poor values for specificity, PPV, and accuracy $(21.3 \%, 19.2 \%$, and $33.3 \%$ respectively). In contrast, similar inquiries using chest $\mathrm{CT}$ as index test, excellent sensitivity, specificity, NPV, and accuracy $(80.0 \%, 86.7 \%, 95.6 \%$, and $85.6 \%$, respectively) were reported, beside a moderate value for PPV (54.5\%).

Conclusion POCUS may provide early ER triage with a useful, rapid, low-threshold, and safe screening tool in evaluating possible COVID-19 infections. Due to limited specificity, suggestive POCUS lung findings should be confirmed with RTPCR or chest CT.
\end{abstract}

Keywords POCUS lung $\cdot$ Emergency room $\cdot$ Sensitivity $\cdot$ Negative predictive value $\cdot$ COVID-19

\section{Introduction}

In December 2019, multiple clusters of patients presenting with pneumonia of unknown cause were reported by Chinese healthcare facilities, which were later on revealed as infections due to a novel betacoronavirus. The identification of the genome of this virus, presently known as severe acute respiratory syndrome coronavirus 2 (SARS-

Nick Narinx

nick.narinx@student.kuleuven.be

1 Faculty of Medicine, KU Leuven, Herestraat 49, 3000 Leuven, Belgium

2 Department of Microbiology, Imelda Hospital, Bonheiden, Belgium

3 Department of Radiology, Imelda Hospital, Bonheiden, Belgium

4 Department of Emergency Medicine, Imelda Hospital, Bonheiden, Belgium
CoV-2), connects it to RNA viruses that caused the severe acute respiratory syndrome (SARS) and Middle East respiratory syndrome (MERS) [1]. Since its appearance on Chinese territory, the virus has spread to over 200 countries and regions in the world, accounting for a global outbreak of coronavirus disease 2019 (COVID-19), ever since posing a serious threat to global healthcare. Although several clinical trials are ongoing in the search for a treatment for COVID19 , an effective approach in the eradication of this virus is yet to be found, limiting current strategies to early detection and isolation of patients [2]. Concerning detection of an active SARS-CoV-2 infection, real-time polymerase chain reaction (RT-PCR) has been postulated the gold standard, with reported sensitivity numbers between 62.5 and $89 \%$ and a specificity up to $98.8 \%[3,4]$. Past infection status and immune response can be complementarily achieved through serologic testing, attaining sensitivity values between 30.1 and $91.4 \%$, depending on the time since onset of first symptoms, and specificity values over 98\% [5]. 
Recently, chest computed tomography (CT) has been suggested as an additional tool for the initial assessment of potentially infected patients, reporting an even higher sensitivity of up to $97 \%[4,6]$. Beside RT-PCR, serologic testing, and chest CT, point-of-care lung ultrasound (POCUS lung) was proposed as a complementary tool in the early diagnosis and follow-up of COVID-19 patients. Studies on clinical manifestations of COVID-19 with POCUS imaging include several findings: typically a variety of B-line patterns (focal, multifocal, and confluent), common thickening of the pleural line, occasional consolidations, and more uncommonly effusions [7-9]. These manifestations on the other hand are not specific for COVID-19 pathology as they have already been described in POCUS of multiple other pathologies involving the lungs [10-12]. An integration of these signs, the BLUE-protocol, developed in 2008, allows swift diagnosis of patients presenting in the emergency department with acute respiratory failure [11]. Notwithstanding this existing protocol and the fact that POCUS is already widely adopted in critical care settings as a useful diagnostic and monitoring tool, current literature however contains few knowledge about its performance in COVID-19 patients [10]. Due to the technique's safety, availability, low cost, absence of radiation, and point-ofcare use, it could be a major and easily accessible utility in the early diagnosis and management of COVID-19 [13]. This retrospective study aims to provide a first analysis of the diagnostic accuracy of POCUS lung used in an emergency setting in the examination of suspected COVID-19 patients. Evaluation of this technique could lead to potential useful insights for its use in early triage in the ER, in the screening of COVID-19 pathology.

\section{Materials and methods}

In writing this report, we adopted the standards for reporting diagnostic accuracy studies (STARD 2015) [14]. This study was found to be in accordance with the Health Insurance Portability and Accountability Act, and the institutional review board of our hospital (Imelda Hospital Bonheiden, Belgium) approved this retrospective study, for which written informed consent was waived. All clinical examinations and tests requiring patient contact, mentioned in this study, were performed by hospital personnel (either physicians or nurses) while mandatorily wearing personal protective equipment (PPE) (at a minimum: FFP2 mask, gloves, goggles or face shield, and medical gown).

\section{Participants}

For this study, 93 patients with an admission to our hospital's emergency department between March 28th and April 20th,
2020, who were suspected of a possible COVID-19 infection and underwent all three diagnostic tests (RT-PCR, chest CT, and POCUS lung) on the same date, were included. Suspicion of a possible COVID-19 infection was assessed following the guidelines drafted by the Belgian authorities [15]. A possible case of COVID-19 was any person presenting with symptoms of an acute infection of the upper or lower airways that was newly appearing or-in patients with chronic respiratory symptoms - got worse. Results of both RT-PCR for SARSCoV-2 and chest CT, as well as age, sex, and symptoms at admission, were extracted from the patients' electronic medical records of our hospital.

\section{SARS-CoV-2 RT-PCR}

For SARS-CoV-2 detection in nasopharyngeal swabs (eSwab, Copan Diagnostics, Brescia, Italy), two PCR methods targeting the E-gene were used. Primers and probe sequences were provided by the Belgian National Reference Center (University Hospital Leuven, Leuven, Belgium). The ARIES system (Luminex, Austin, USA) is an all-in-one system, performing extraction, purification, amplification, and detection in one run. Analyzation of PCR results was performed with Luminex SYNCT software. A second platform is the Rotorgene Q (Qiagen, Hilden, Germany). NucliSENS EasyMAG (BioMérieux, Marcy l'Étoile, France) was used to perform extraction. Interpretation of data was managed with Rotor-Gene Q Series software. Utilizing a dilution series of inactivated SARS-CoV-2, sensitivity of Aries was analyzed. Rotorgene Q platform sensitivity was validated compared with Aries. Cross reactivity for other human coronaviruses, influenza or respiratory syncytial virus (RSV) has not been demonstrated [16].

\section{Chest CT protocol and analysis}

Chest $\mathrm{CT}$ and analysis were performed by two cardiothoracic radiologists (with 8 and 7 years of cardiothoracic imaging experience) of Imelda Hospital Bonheiden, in accordance with their recently published protocol [16]. Low-dose chest CT for all patients was performed by using the Somatom Definition AS 64-slice 0.6-mm detector scanner (Siemens Healthineers, Forchheim, Germany), setting reference values to $100 \mathrm{kVp}$ and $20 \mathrm{mAs}$ with a pitch of 1.2 and $0.5 \mathrm{~s}$ gantry rotation time. Motion artifacts in dyspneic COVID-19 patients were limited using a relatively high pitch. Images were reconstructed at $1 \mathrm{~mm}$ slice thickness and $0.7 \mathrm{~mm}$ increment with a standard lung-tissue kernel (I50f medium sharp) and at $3 \mathrm{~mm}$ slice thickness and $3 \mathrm{~mm}$ increment with a standard soft tissue kernel (I31f medium smooth) using sinogram-affirmed iterative reconstruction (SAFIRE) strength 3. Image reconstructions were achieved with a FOV of $450 \mathrm{~mm}$ and a matrix size of $512 \times 512$ pixels. 
Next, chest CT images were analyzed and, blinded to the RT-PCR results, scored as suggestive for or inconsistent with COVID-19 infection based on the presence of clinical manifestations as presented by $\mathrm{Ng}$ et al. and Shi et al. [17, 18]. Additionally, extent of pulmonary involvement was estimated using a semiquantitative scoring system as reported previously $[19,20]$.

\section{POCUS lung protocol}

Upon a patient's admission to the emergency department, firstly, clinical triage was conducted by a triage nurse. Patients were subsequently evaluated by an emergency medical doctor (EMD) to assess the possibility of a COVID-19 infection. Assessment by the triage nurse and the EMD was managed according to the triage protocol reported by Zhang et al. [21]. If suspected for COVID-19, POCUS of both lungs was performed by an EMD within 30 min of admission, before either RT-PCR or chest CT was carried out. POCUS was conducted by one of three EMDs, all certified for lung ultrasound (US) and each having more than 5 years of US experience. POCUS was achieved in the emergency department using owned equipment, the Philips Sparq ultrasound system equipped with a C5-1 broadband curved array $(5 \mathrm{MHz}$, depth $8 \mathrm{~cm})$. The examination was executed in accordance with the BLUE protocol as designed by Lichtenstein et al. [10-12, 22]. Three BLUE points for each hemithorax were scanned in supine (PLAPS point) or semi-recumbent position (upper and lower BLUE point). All 6 BLUE points were examined for B-line patterns and thickening of the pleural line, being typical US findings in COVID-19 [7-9]. POCUS lung reports were drawn up in the patient's medical records, stating a dichotomous value for each of the manifestations per BLUE point. A B-line pattern was considered present if more than $3 \mathrm{~B}$-lines could be found.

\section{POCUS lung analysis}

All POCUS lung reports were analyzed and evaluated as suggestive for or inconsistent with COVID-19 infection. Considering that conclusive reports about the diagnosis of COVID-19 by use of US are still to be published, a solid evaluation and scoring system is not yet available. For our analysis, we only took into account the B-line parameter, being the most reported clinical manifestation. We then considered POCUS lung positive if one or more BLUE points showed a positive B-line parameter. Written POCUS lung reports were analyzed by the researcher, blinded to all clinical information, US imaging, RT-PCR, and chest CT test results.

\section{Statistical analysis}

The statistical analysis required for this study was performed using RStudio version 1.2.5042 (RStudio, Inc., Boston, MA). Performance of both index tests, POCUS lung and chest CT, was computed in terms of sensitivity, specificity, positive predictive value (PPV), negative predictive value (NPV), and accuracy with 95\% confidence interval (CI) using the reportROC package. RT-PCR for SARS-CoV-2 served as the reference test in these calculations. Demographical patient data was tested for normal distribution by usage of the Shapiro-Wilk test. Summary statistics for age are reported as means \pm standard deviations (SD), and sex is reported as a fixed proportion. The Student $t$ test for independent samples was used to compare continuous variables between groups. The two- or three-proportions Z-test was used to compare proportions between groups. $p<0.05$ was considered to indicate a statistically significant difference.

\section{Results}

\section{General description}

Three patients were excluded from analysis due to not receiving all three tests (RT-PCR, chest CT, and POCUS lung) on the same date (i.e. date of admission to the ER). After exclusion of these patients, 90 patients remained for further analysis. Mean age for all patients included in the study was $50.4 \pm$ 16.3 years (SD). Comparing the mean age of COVID-19positive patients $(49.9 \pm 13.7$ years $)$ with the mean age of COVID-19-negative patients ( $50.5 \pm 16.9$ years), we calculated no significant difference $(p=0.907)$ between the two groups. With respect to sex of the study population, $45.6 \%$ (41/90) of all study subjects are of male sex. Comparison of the proportions of males in COVID-19-positive patients and COVID-19-negative patients $(60.0 \%(9 / 15)$ vs $42.7 \%$ (32/ $75)$ ) showed no significant difference $(p=0.344)$. At admission, the most common clinical symptoms where cough $(52.2 \%(47 / 90))$ and dyspnea $(51.1 \%(46 / 90))$, with no significant difference between COVID-19-positive and negative patients $(p=0.706$ and $p=1.000)$. Patient demographics and admission symptoms are summarized in Table 1.

\section{Diagnostic accuracy of POCUS lung and chest CT in diagnosing COVID-19}

Figure 1 shows the flowchart of the study, comparing RTPCR for the detection of SARS-CoV-2, designated as reference standard, to the index test, POCUS lung. The performance of POCUS lung comprises outstanding sensitivity and NPV (93.3\% (14/15) and 94.1\% (16/17), respectively) while showing poor values for specificity, PPV, and accuracy (21.3\% (16/75), $19.2 \%$ (14/73), and 33.3\%, respectively). In 
Table 1 Study population demographics

\begin{tabular}{lllll}
\hline & All $(n=90)$ & COVID-19+ $(n=15)$ & COVID-19- $(n=75)$ & $p$ value \\
\hline $\begin{array}{l}\text { Demographics } \\
\text { Age (year) }\end{array}$ & $50.4 \pm 16.3$ & $49.9 \pm 13.7$ & & \\
Male sex* & $41(45.6)$ & $9(60.0)$ & $50.5 \pm 16.9$ & 0.907 \\
Clinical symptoms at ER admission & & & 0.344 \\
Fever* & $24(26.7)$ & $5(33.3)$ & $19(25.3)$ & \\
Cough* & $47(52.2)$ & $9(60.0)$ & $38(50.7)$ & 0.749 \\
Dyspnea* & $46(51.1)$ & $8(53.3)$ & $38(50.7)$ & 0.706 \\
Chest pain* & $25(27.8)$ & $1(0.07)$ & $24(0.32)$ & 1.000 \\
\hline
\end{tabular}

Age data are means \pm SD

*Data are numbers of patients, with percentages in parentheses contrast, similar inquiries using chest CT as index test, shown in Fig. 2, provided excellent sensitivity, specificity, NPV, and accuracy $(80.0 \%(12 / 15), 86.7 \%$ (65/75), 95.6\% (65/68), and $85.6 \%$, respectively), beside an only moderate value for PPV (54.5\% (12/22)). Results for the diagnostic accuracy of POCUS lung and chest CT, both compared with RT-PCR, are summarized in Table 2. Further, Table 3 shows POCUS lung findings correlated to $\mathrm{CT}$ results, both pooled and separately for each EMD. Pooled performance result of all three EMDs shows an agreement of POCUS lung with CT results in 39 out of 90 cases $(43.3 \%)$. For each EMD separately, the POCUS lung-CT agreement was 15/46 (32.6\%), 15/25 $(60.0 \%)$, and $9 / 19(47.4 \%)$, respectively, with a $p$ value of 0.08 for inter-EMD proportions. In addition, Fig. 3 depicts clinical findings using POCUS lung and chest CT in a clinically suspected and RT-PCR-confirmed COVID-19-positive patient. Concerning clinical findings in RT-PCR-confirmed COVID-19-positive patients $(n=15)$, position of lung infiltrate was evaluated, comparing POCUS lung imaging with CT imaging. In 13 cases, CT protocol reported an involvement of both entire lungs (theoretically corresponding to all 6 BLUE-points), while only 2 of those 13 cases (15.4\%) were evaluated as entire lung involvement by means of POCUS lung (6 BLUE-points). In all other 11 cases, POCUS lung reported positive BLUE points in a range from 1 to 5 . Of the remaining 2 cases, one was negatively protocolled for COVID-19 by CT, while POCUS lung withheld a positive result and the other case vice versa.

\section{Discussion}

The use of point-of-care lung ultrasound could play a beneficial role in the screening of patients with a suspected COVID19 infection, when integrated in the early triage in the emergency department. In comparison with RT-PCR, POCUS lung delivers an excellent sensitivity and NPV, 93.3\% and 94.1\%, respectively, labelling the technique a useful screening test. Other values of performance covered in this study, specificity, PPV, and accuracy $(21.3 \%, 19.2 \%$, and $33.3 \%$, respectively), however, state its limitations in achieving an accurate and specific diagnosis. The low values for specificity, PPV, and accuracy were expected as we hypothesized that a POCUS lung could be considered positive whenever one or more out of six BLUE points were positive and that one BLUE point is treated positive when a B-line pattern surfaced. As confirmed in previous studies, B-lines as a sole entity are unspecific and fit in the ultrasound diagnostic landscape of multiple pathologies as well as the senile lung [10, 11, 22, 23]. At present time, there are no studies available that would allow us to make a comparison to confirm whether or not the accuracy of our analysis is representable. However, in order to evaluate whether or not POCUS lung results could be reproduced in a consistent way, inter-EMD observations were investigated and showed a nonsignificant result ( $p$ value of 0.08 ) for POCUS-CT agreement. Although this $p$ value points in the direction of a reproducible test with good POCUS-CT correlation, the argument is not very convincing, for which we

Table 2 Diagnostic accuracy of POCUS lung and chest CT in the diagnosis of COVID-19 infection

\begin{tabular}{|c|c|c|c|c|c|c|c|c|c|}
\hline & \multicolumn{4}{|c|}{ Results $(n)$} & \multicolumn{5}{|c|}{ Test performance (\% with $95 \% \mathrm{CI}$ ) } \\
\hline & $\mathrm{TP}$ & $\mathrm{TN}$ & FP & FN & Sensitivity & Specificity & PPV & NPV & Accuracy \\
\hline POCUS lung $(n=90)$ & 14 & 16 & 59 & 1 & $93.3(80.7-100.0)$ & $21.3(12.1-30.6)$ & $19.2(10.1-28.2)$ & $94.1(82.9-100.0)$ & $33.3(32.8-33.8)$ \\
\hline Chest CT $(n=90)$ & 12 & 65 & 10 & 3 & $80.0(59.8-100.0)$ & $86.7(79.0-94.4)$ & $54.5(33.7-75.4)$ & $95.6(90.7-100.0)$ & $85.6(85.3-85.8)$ \\
\hline
\end{tabular}

$T P$ true positive, $T N$ true negative, $F P$ false positive, $F N$ false negative, $P P V$ positive predictive value, $N P V$ negative predictive value 
Table 3 Pooled and inter-EMD correlation between POCUS lung and CT results

\begin{tabular}{|c|c|c|c|c|c|c|}
\hline \multirow[t]{2}{*}{ EMD } & \multirow{2}{*}{$\begin{array}{l}\text { Number of POCUS } \\
\text { lung performed }\end{array}$} & \multirow[t]{2}{*}{ POCUS result } & \multicolumn{2}{|c|}{$\mathrm{CT}$ protocol result } & \multirow[t]{2}{*}{ POCUS lung vs CT agreement } & \multirow[t]{2}{*}{$p$ value } \\
\hline & & & Positive & Negative & & \\
\hline \multirow[t]{2}{*}{ All EMDs } & \multirow[t]{2}{*}{$90(100.0 \%)$} & Positive & 22 & 51 & \multirow[t]{2}{*}{$39 / 90(43.3 \%)$} & \\
\hline & & Negative & 0 & 17 & & \\
\hline \multirow[t]{2}{*}{ EMD \#1 } & \multirow[t]{2}{*}{$46(51.1 \%)$} & Positive & 9 & 31 & \multirow[t]{2}{*}{$15 / 46(32.6 \%)$} & \multirow[t]{6}{*}{0.08} \\
\hline & & Negative & 0 & 6 & & \\
\hline \multirow[t]{2}{*}{ EMD \#2 } & \multirow[t]{2}{*}{$25(27.8 \%)$} & Positive & 8 & 10 & \multirow[t]{2}{*}{$15 / 25(60.0 \%)$} & \\
\hline & & Negative & 0 & 7 & & \\
\hline \multirow[t]{2}{*}{ EMD \#3 } & \multirow[t]{2}{*}{$19(21.1 \%)$} & Positive & 5 & 10 & \multirow[t]{2}{*}{$9 / 19(47.4 \%)$} & \\
\hline & & Negative & 0 & 4 & & \\
\hline
\end{tabular}

Data are numbers of performed tests, with percentages in parentheses

$E M D$ emergency medical doctor

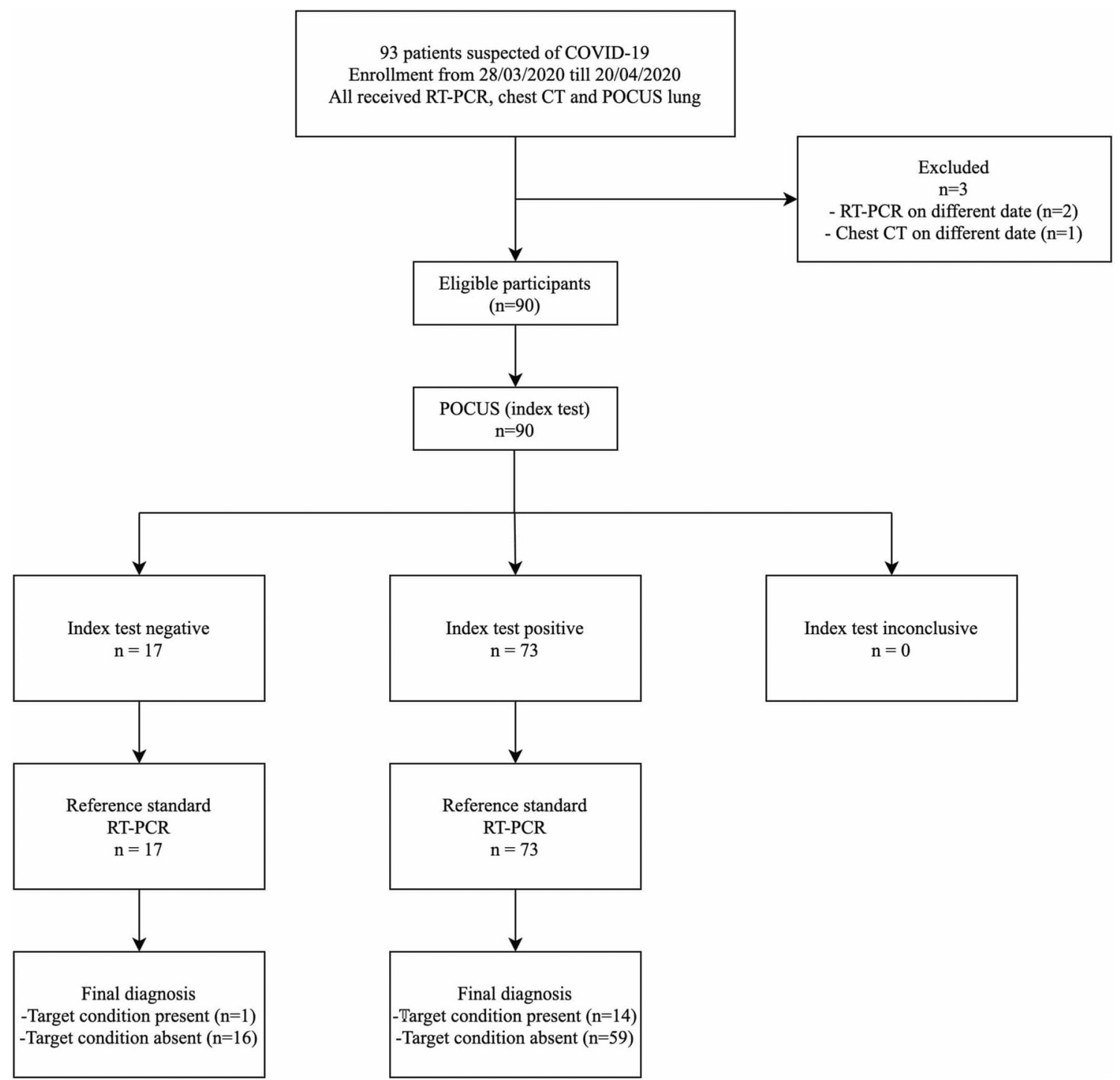

Fig. 1 Study flowchart for POCUS lung as index test. RT-PCR real-time polymerase chain reaction, chest CT chest computed tomography, POCUS point-of-care ultrasound 


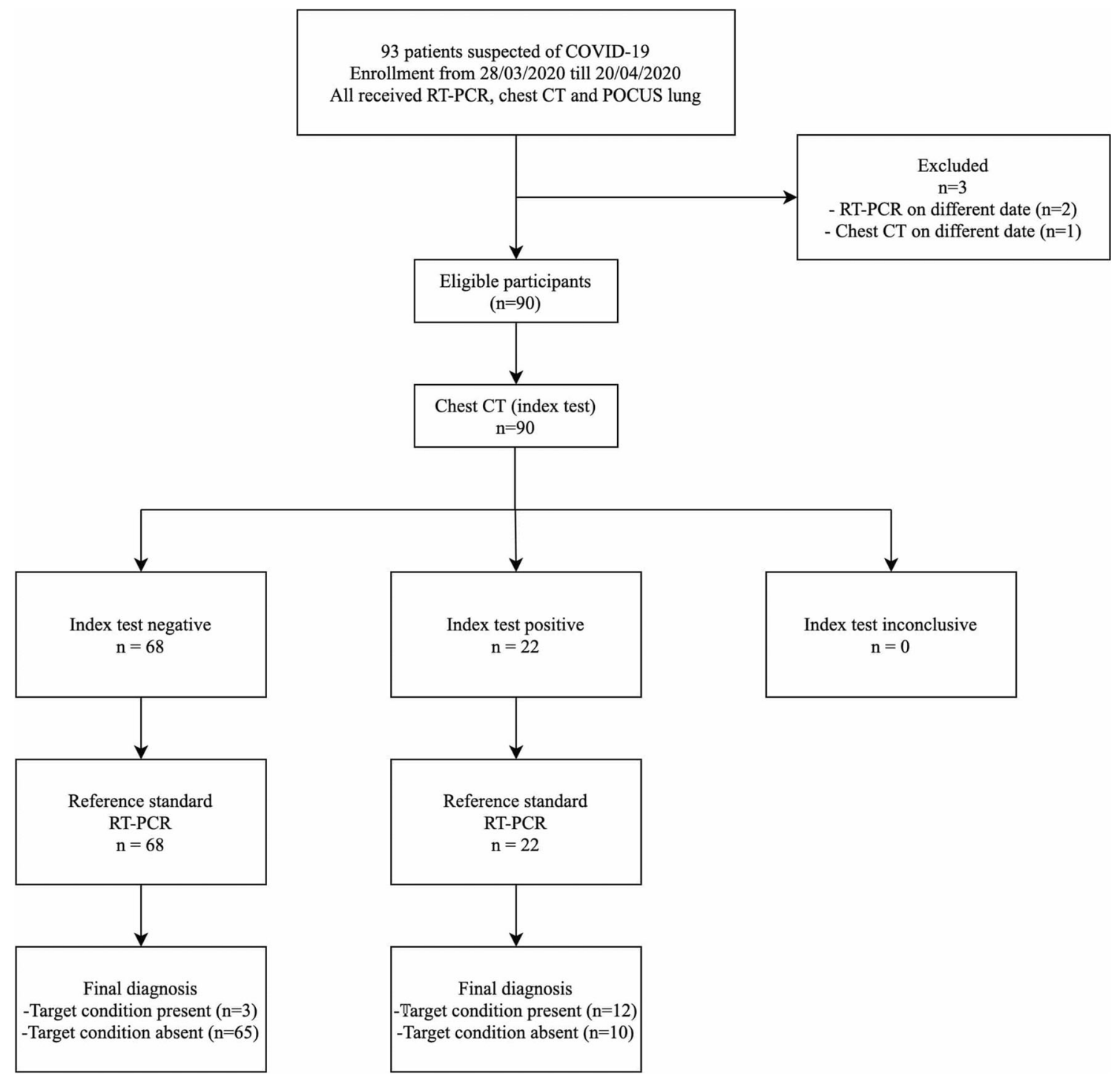

Fig. 2 Study flowchart for chest CT as index test. RT-PCR real-time polymerase chain reaction, chest CT chest computed tomography, POCUS pointof-care ultrasound

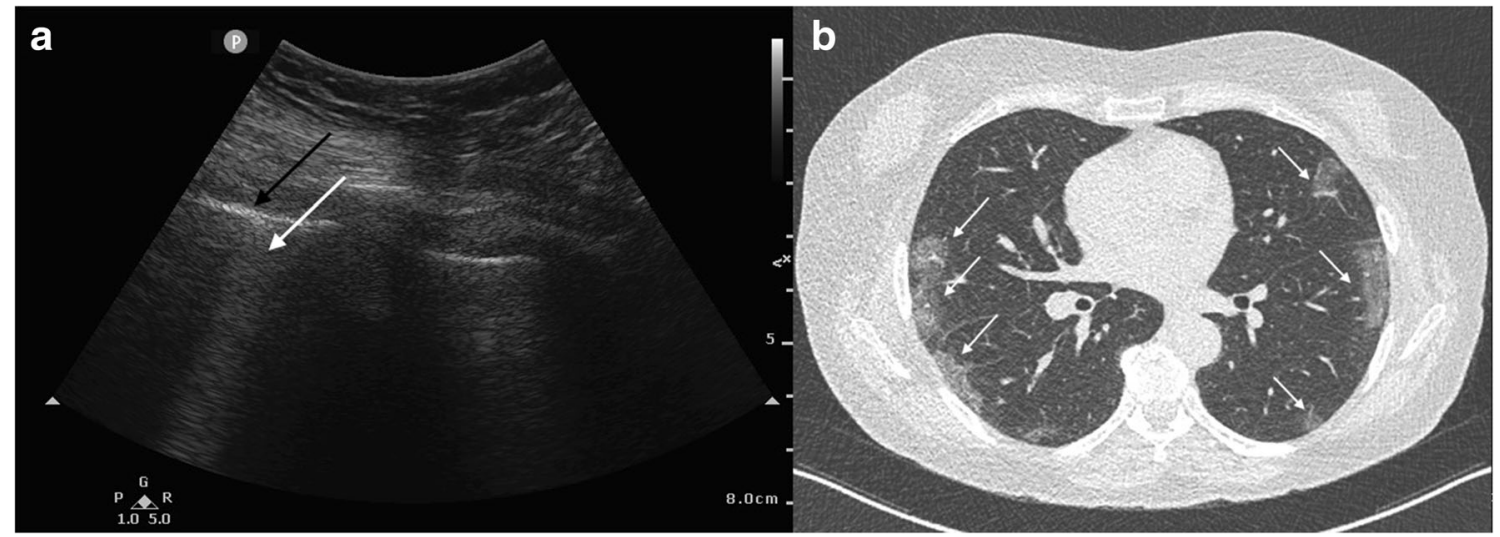

Fig. 3 Example POCUS lung and CT images in one RT-PCR-confirmed COVID-19-positive patient. Images were obtained from a 66-year-old woman presenting in the ER with dyspnea, coughing, and fever for 8 days. POCUS lung image of the right PLAPS point (a) shows the pleural line (black arrow) as well as a confluent pattern of B-lines (white arrow). Axial CT (b) shows typical early COVID-19 findings with bilateral subpleural areas of ground-glass opacities (white arrows). 
consider sample size of our study the root cause of this issue. Nonetheless, the acknowledgement that POCUS lung could be used as a proper screening test is of valuable information, provided it can be performed with the right safety precautions using PPE. Implementation in early triage in the ER could be useful as it meets the priority objective of the ER of saving time in the diagnostic approach of patients.

Additionally, parallel investigation of chest CT performance compared with RT-PCR, within the same pool of patients, provided excellent sensitivity, specificity, NPV, and accuracy $(80.0 \%, 86.7 \%, 95.6 \%$, and $85.6 \%$, respectively), beside an only moderate value for PPV (54.5\%). These results are in line with results of low-dose chest CT recently published by Dangis et al. [16]. Altogether, the results demonstrated for both POCUS lung and chest CT designate the two techniques as a complementary pair. While having the ability to rule out COVID-19 lung infection using POCUS lung as a screening test, subsequent chest $\mathrm{CT}$ could provide a more confident diagnosis and allow for assessment of the extent of lung involvement [16]. Distilling a clinical decision rule for COVID-19 involvement based on this study would depend on the clinical assessment made by the physician with regard to patient profile and severity of symptoms. In asymptomatic patients, or patients presenting with only mild symptoms of an airway infection, POCUS lung could be the designated screening test. A negative POCUS lung result could then justify ruling out a COVID-19 infection. On the other hand, highrisk patients (e.g. cardiovascular or pulmonary issues in the medical history) and patients presenting with more severe lung pathology would benefit more from receiving CT imaging either confirming COVID-19 infection or ruling it out and at the same time providing more information on other possible etiologies.

Apart from no radiation exposure, POCUS lung has numerous other advantages making it a considerable technique for screening in early triage in the ER. Low cost, high availability, bedside testing, and time between exam and result are the other main benefits, but also low threshold for testing vulnerable patients (e.g. children, pregnant women) and the possibility of using it under sterile coverage or being able to disinfect quickly after use make it an outstanding screening tool [13].

This study has several limitations. First of all, this retrospective study was subject to an inherent selection bias. Secondly, study participants were included providing they had an admission to the ER, making these potentially more severe cases than seen in general practice. However, every patient showing one or more symptoms of an upper or lower respiratory tract infection, as laid out by the Belgian authorities, was included. In extension, thirdly, notwithstanding clinical manifestations associated with a respiratory tract infection (e.g. fever, cough, and dyspnea) are the most common symptoms; this study did not include any subjects with non-pulmonary signs (e.g. headache, nausea, diarrhea, and vomiting) of a COVID-19 infection [24].
A fourth limitation that can have an impact on the results of this study is timing. The time window in which participants were included was between March 28th and April 20th, 2020, situating it behind the peak of the infection in Belgium. This resulted in a relatively small sample size $(n=93)$. Furthermore, the test population included contained only a small number of participants who eventually tested positively for SARS-CoV-2 by RT-PCR ( $n=15 ; 16.1 \%$ of the total study population). A next limitation, and perhaps the most important one, is the lack of a guideline for POCUS lung in COVID-19. The criterion to consider a POCUS lung as suggestive for COVID-19 by one positive BLUE point presenting with a B-line pattern is not evidence-based. Current literature on the other hand contains no evidence on the matter so far. By altering this hypothesis, we expect that reported values for diagnostic accuracy could differ.

Aside from preceding arguments, we acknowledge the fact that ER medical doctors are differently qualified than radiologists in context of performing ultrasound. Despite their skills and training in ultrasound, the technique remains strongly researcher-dependent and results could vary accordingly. This applies equally to the three EMDs who actually performed the POCUS lung. Interpersonal results could be subject to variation as it is an inherent disadvantage of the technique. Finally, the use of a highly sensitive RT-PCR assay does not guarantee its precision. Despite having a negative RT-PCR test, a patient showing typical symptoms combined with a positive POCUS lung or chest CT may still be COVID-19 infected. Extended research is needed to exclude an infection isolated to the lower regions of the respiratory tract.

In conclusion, point-of-care lung ultrasound may provide early triage in the ER with a useful, rapid, lowthreshold, and safe screening tool in the evaluation of a possible COVID-19 infection. Particular benefits of the technique are its high sensitivity and negative predictive value, indicating a high level of confidence that a negative POCUS in symptomatic patients is a true result, preventing them from receiving more invasive, cost-intensive, and time-consuming tests.

\section{Compliance with ethical standards}

Conflict of interest The authors declare that they have no conflict of interest.

Ethics approval This research study was conducted retrospectively from data obtained for clinical purposes. We consulted extensively with the IRB of Imelda Hospital, Bonheiden, Belgium, who determined that our study did not need ethical approval. An IRB official waiver of ethical approval was granted from the IRB of Imelda Hospital, Bonheiden, Belgium.

Consent to participate/publish The need for written informed consent was waived by the IRB of Imelda Hospital, Bonheiden, Belgium. 


\section{References}

1. Abduljalil JM, Abduljalil BM (2020) Epidemiology, genome, and clinical features of the pandemic SARS-CoV-2: a recent view. New Microbes New Infect 35:100672. https://doi.org/10.1016/j.nmni. 2020.100672

2. Tu Y-F, Chien C-S, Yarmishyn AA, Lin YY, Luo YH, Lin YT, Lai WY, Yang DM, Chou SJ, Yang YP, Wang ML, Chiou SH (2020) A review of SARS-CoV-2 and the ongoing clinical trials. Int J Mol Sci 21. https://doi.org/10.3390/ijms21072657

3. Zitek T (2020) The appropriate use of testing for COVID-19. West J Emerg Med 21:470-472. https://doi.org/10.5811/westjem.2020. 4.47370

4. Kim H, Hong H, Yoon SH (2020) Diagnostic performance of CT and reverse transcriptase-polymerase chain reaction for coronavirus disease 2019: a meta-analysis. Radiology 201343:E145-E155. https://doi.org/10.1148/radiol.2020201343

5. Deeks JJ, Dinnes J, Takwoingi Y et al (2020) Antibody tests for identification of current and past infection with SARS-CoV-2. Cochrane Database Syst Rev 6:CD013652. https://doi.org/10. 1002/14651858.CD013652

6. Ai T, Yang Z, Hou H, Zhan C, Chen C, Lv W, Tao Q, Sun Z, Xia L (2020) Correlation of chest CT and RT-PCR testing in coronavirus disease 2019 (COVID-19) in China: a report of 1014 cases. Radiology 200642:E32-E40. https://doi.org/10.1148/radiol. 2020200642

7. Lomoro P, Verde F, Zerboni F, Simonetti I, Borghi C, Fachinetti C, Natalizi A, Martegani A (2020) COVID-19 pneumonia manifestations at the admission on chest ultrasound, radiographs, and CT: single-center study and comprehensive radiologic literature review. Eur J Radiol Open 7:100231. https://doi.org/10.1016/j.ejro.2020. 100231

8. Peng Q-Y, Wang X-T, Zhang L-N, Chinese Critical Care Ultrasound Study Group (CCUSG) (2020) Findings of lung ultrasonography of novel corona virus pneumonia during the 20192020 epidemic. Intensive Care Med 46:849-850. https://doi.org/ 10.1007/s00134-020-05996-6

9. Buonsenso D, Piano A, Raffaelli F et al (2020) Point-of-Care Lung Ultrasound findings in novel coronavirus disease-19 pnemoniae: a case report and potential applications during COVID-19 outbreak. Eur Rev Med Pharmacol Sci 24:2776-2780. https://doi.org/10. 26355/eurrev_202003_20549

10. Mojoli F, Bouhemad B, Mongodi S, Lichtenstein D (2019) Lung ultrasound for critically ill patients. Am J Respir Crit Care Med 199: 701-714. https://doi.org/10.1164/rccm.201802-0236CI

11. Lichtenstein DA, Mezière GA (2008) Relevance of lung ultrasound in the diagnosis of acute respiratory failure: the BLUE protocol. Chest 134:117-125. https://doi.org/10.1378/chest.07-2800

12. Bekgoz B, Kilicaslan I, Bildik F, Keles A, Demircan A, Hakoglu O, Coskun G, Demir HA (2019) BLUE protocol ultrasonography in Emergency Department patients presenting with acute dyspnea. Am J Emerg Med 37:2020-2027. https://doi.org/10.1016/j.ajem. 2019.02 .028
13. Abu-Zidan FM (2012) Point-of-care ultrasound in critically ill patients: where do we stand? J Emerg Trauma Shock 5:70-71. https:// doi.org/10.4103/0974-2700.93120

14. Bossuyt PM, Reitsma JB, Bruns DE, Gatsonis CA, Glasziou PP, Irwig L, Lijmer JG, Moher D, Rennie D, de Vet HCW, Kressel HY, Rifai N, Golub RM, Altman DG, Hooft L, Korevaar DA, Cohen JF (2015) STARD 2015: an updated list of essential items for reporting diagnostic accuracy studies. BMJ 351:h5527. https://doi.org/10. 1136/bmj.h5527

15. Sciensano COVID-19 - Gevalsdefinitie en testing | Coronavirus Covid-19. In: Covid-19sciensanobe. https://covid-19.sciensano.be/ nl/covid-19-gevalsdefinitie-en-testing. Accessed 15 April 2020

16. Dangis A, Gieraerts C, Bruecker YD, Janssen L, Valgaeren H, Obbels D, Gillis M, Ranst MV, Frans J, Demeyere A, Symons R (2020) Accuracy and reproducibility of low-dose submillisievert chest CT for the diagnosis of COVID-19. Radiol Cardiothorac Imaging 2:e200196. https://doi.org/10.1148/ryct.2020200196

17. Ng M-Y, Lee EY, Yang J et al (2020) Imaging profile of the COVID-19 infection: radiologic findings and literature review. Radiol Cardiothorac Imaging 2:e200034. https://doi.org/10.1148/ ryct.2020200034

18. Shi H, Han X, Jiang N, Cao Y, Alwalid O, Gu J, Fan Y, Zheng C (2020) Radiological findings from 81 patients with COVID-19 pneumonia in Wuhan, China: a descriptive study. Lancet Infect Dis 20:425-434. https://doi.org/10.1016/S1473-3099(20)30086-4

19. Inui S, Fujikawa A, Jitsu M, Kunishima N, Watanabe S, Suzuki Y, Umeda S, Uwabe Y (2020) Chest CT findings in cases from the cruise ship "diamond princess" with coronavirus disease 2019 (COVID-19). Radiol Cardiothorac Imaging 2:e200110. https:// doi.org/10.1148/ryct.2020200110

20. Pan F, Ye T, Sun P, Gui S, Liang B, Li L, Zheng D, Wang J, Hesketh RL, Yang L, Zheng C (2020) Time course of lung changes on chest CT during recovery from 2019 novel coronavirus (COVID-19) pneumonia. Radiology 200370:715-721. https://doi. org/10.1148/radiol.2020200370

21. Zhang J, Zhou L, Yang Y, Peng W, Wang W, Chen X (2020) Therapeutic and triage strategies for 2019 novel coronavirus disease in fever clinics. Lancet Respir Med 8:e11-e12. https://doi.org/10. 1016/S2213-2600(20)30071-0

22. Miller A (2015) Practical approach to lung ultrasound. BJA Educ 16:39-45. https://doi.org/10.1093/bjaceaccp/mkv012

23. Chiesa AM, Ciccarese F, Gardelli G, Regina UM, Feletti F, Bacchi Reggiani ML, Zompatori M (2015) Sonography of the normal lung: comparison between young and elderly subjects. J Clin Ultrasound JCU 43:230-234. https://doi.org/10.1002/jcu.22225

24. Li L-Q, Huang T, Wang Y-Q, Wang ZP, Liang Y, Huang TB, Zhang HY, Sun W, Wang Y (2020) COVID-19 patients' clinical characteristics, discharge rate, and fatality rate of meta-analysis. J Med Virol 92:577-583. https://doi.org/10.1002/jmv.25757

Publisher's note Springer Nature remains neutral with regard to jurisdictional claims in published maps and institutional affiliations. 